\title{
Are symptoms of anxiety and depression associated with colorectal screening perceptions and behaviors among older adults in primary care?
}

\author{
Hind A. Beydoun ${ }^{1}$, Suraj Khanal ${ }^{1}$, May A. Beydoun ${ }^{2}$, Alan B. Zonderman², \\ Ravinder Mohan ${ }^{3}$, Agatha Parks-Savage ${ }^{4}$ \\ ${ }^{1}$ Graduate Program in Public Health, Eastern Virginia Medical School, Norfolk, USA; \\ baydouha@evms.edu, khanalsuraj@gmail.com \\ ${ }^{2}$ National Institute on Aging, Baltimore, USA; baydoum@mail.nih.gov, zondermana@mail.nih.gov \\ ${ }^{3}$ Department of Family Medicine, Eastern Virginia Medical School, Norfolk, USA; docmohaan@gmail.com \\ ${ }^{4}$ Office of Graduate Medical Education, Eastern Virginia Medical School, Norfolk, USA; parksac@evms.edu
}

Received 25 December 2013; revised 27 January 2014; accepted 11 February 2014

Copyright (C 2014 Hind A. Beydoun et al. This is an open access article distributed under the Creative Commons Attribution License, which permits unrestricted use, distribution, and reproduction in any medium, provided the original work is properly cited. In accordance of the Creative Commons Attribution License all Copyrights (C) 2014 are reserved for SCIRP and the owner of the intellectual property Hind A. Beydoun et al. All Copyright (C) 2014 are guarded by law and by SCIRP as a guardian.

\section{ABSTRACT}

Despite the existence of colorectal cancer (CRC) screening guidelines, population-based studies have consistently shown under-utilization of CRC screening procedures among older adults in the United States. We examined whether symptoms of anxiety and depression are associated with colorectal cancer (CRC) screening perceptions and behaviors among older adults in a primary care setting. A cross-sectional study was conducted by using a sample of 143 family medicine patients who completed an 88-item anonymous self-administered questionnaire covering symptoms of anxiety and depression as well as CRC screening perceptions (defined based on the Health Belief Model) and behaviors (defined as ever use of or adherence to CRC testing). Moderate-to-clinically significant anxiety and depressive symptoms were, respectively, prevalent in $47 \%$ and $42 \%$ of participants. Perceived benefits and barriers were the only Health Belief Model constructs associated with anxiety. Perceived barriers were positively associated with anxiety symptoms after adjustment for confounders, including age, gender, race/ ethnicity, marital status, education, smoking history, body mass index and self-rated health. By contrast, perceived benefits were negatively associated with anxiety symptoms only in the unadjusted model. Neither anxiety nor depression was associated with ever use of or adherence to CRC testing. Symptoms of anxiety, but not depression, may potentially influence CRC screening perceptions, with implications for behavioral interventions targeting CRC testing.

\section{KEYWORDS}

Anxiety; Behavior; Colorectal Cancer; Depression; Health Belief Model; Perception; Screening

\section{INTRODUCTION}

Colorectal cancer (CRC) is the second leading cause of cancer mortality, accounting for nearly $11 \%$ of all cancer deaths, and the fourth most frequently diagnosed cancer in older adults living in the United States [1]. CRC mortality and incidence rates rank second to lung cancer in men and third after lung cancer and breast cancer among women [2]. In the United States, the lifetime risk for CRC is 1 in 18 or close to $6 \%$ [3,4]. Over $90 \%$ of CRC occurs in individuals above 50 years of age and $75 \%$ result from benign, adenomatous polyps among "average-risk" older adults [4,5]. CRC survival ranges between 94\% among patients with localized CRC and 9\% among patients with metastatic CRC [4].

CRC screening is a cost-effective means of reducing incidence and mortality from CRC through prevention, early detection and treatment of CRC [6,7]. The American Cancer Society (ACS), the National Cancer Institute (NCI) and the United States Preventative Services Task 
Force (USPSTF) agree on the importance of CRC screening $[4,8]$. However, specific recommendations for CRC screening are complex due to the availability of a wide range of CRC screening options [9,10]. Current practice favors one colonoscopy every 10 years as the preferred screening option for “average-risk" older adults, 50 years and older [9]. Alternatively, annual fecal occult blood testing (FOBT) and/or sigmoidoscopy every 5 years are recommended for these "average-risk" older adults [9]. More frequent CRC screening tests are recommended for "intermediate" and "high-risk" individuals [4,11]. Each CRC screening modality has a different risk-benefit profile, with colonoscopy having the most risks and benefits [12]. Moreover, colonoscopy and sigmoidoscopy are collectively referred to as "lower endoscopy" (LE) and are generally perceived as being more invasive than FOBT [13-17].

Despite the existence of CRC screening guidelines, population-based studies, including the Behavioral Risk Factor Surveillance System (BRFSS) and the National Health Interview Survey (NHIS), have consistently shown under-utilization of CRC screening procedures among older adults in the United States [18,19]. The suboptimal use of CRC screening services has been compounded by wide gender, racial/ethnic and socioeconomic disparities in CRC screening behaviors. Therefore, innovative behavioral interventions are needed to increase the proportion of age-eligible individuals who are likely to use CRC screening tests and adhere to CRC screening guidelines [20,21]. A prerequisite to the design, implementation and evaluation of behavioral interventions is a better understanding of the main factors that can influence CRC screening behaviors among older adults in the United States [10,22,23]. The Health Belief Model (HBM) is a commonly used theoretical framework for identifying factors that can influence CRC screening behaviors [24-26], including perceived susceptibility (belief regarding chance of getting a condition), perceived severity (belief in seriousness of condition), perceived benefits (beliefs concerning the effectiveness of taking a particular action in reducing illness threat or producing other desirable outcomes), perceived barriers (beliefs about the negative aspects of taking a particular action) and self-efficacy (one's beliefs about their ability to successfully accomplish the specified behavior). These HBM constructs may be conceptualized as CRC screening perceptions which could have an influence on CRC screening behaviors, including ever use of CRC screening services and adherence to CRC screening guidelines. Therefore, it is important to examine whether individual characteristics that have previously been identified as predictors of CRC screening behaviors can also predict these HBM constructs [27].

A growing body of the literature has identified a po- tential link between CRC screening behaviors and symptoms of anxiety and depression [28-45]. However, no studies have assessed whether CRC screening perceptions which can influence CRC screening behaviors are also linked to symptoms of anxiety and depression. Although evidence supporting this hypothesis remains inconclusive, recent studies have suggested that mental health problems may be implicated in the underuse of cancer screening services $[37,42,43]$. It is plausible that individuals who are diagnosed with a mental illness will likely experience numerous health concerns that can preclude timely and appropriate cancer screening. It is also plausible that ever users of CRC screening who are not adherent with CRC screening guidelines may exhibit more anxiety or depressive symptoms than those who are adherent to these guidelines. Similarly, irrespective colorectal cancer screening behaviors, colorectal cancer screening perceptions or HBM constructs may be influenced by symptoms of anxiety or depression. The purpose of this study is to examine whether symptoms of anxiety and depression are associated with CRC screening perceptions and behaviors among older adults in a primary care setting. We hypothesize that symptoms of anxiety and depression may affect CRC screening perceptions as well as the use of CRC screening services, as delineated by the conceptual model presented in Figure 1.

\section{MATERIALS AND METHODS}

\subsection{Population and Procedures}

A cross-sectional study was conducted between February and September of 2011, using a convenience sample of family medicine patients. Data were collected using an anonymous self-administered questionnaire. A trained research assistant identified age (50 years and older) and language (proficient in English) eligible patients at one family medicine practice and invited them to participate in the study. A packet containing a cover letter and the self-administered questionnaire were provided to these eligible patients. The cover letter included information on study purpose, eligibility criteria, and ethical issues of privacy, confidentiality, risks and benefits as well as instructions on how to contact the principal investigator or the human subject protection office, and how to complete the self-administered questionnaire. Patients who agreed to participate in the study were instructed to complete the self-administered questionnaire on-site or return the completed questionnaire in a selfaddressed and stamped envelope. Alternatively, the research assistant instructed the patients on how to access the web-based version of the self-administered questionnaire, which was developed using QuestionPro (http://www.questionpro.com/). The Institutional Review 


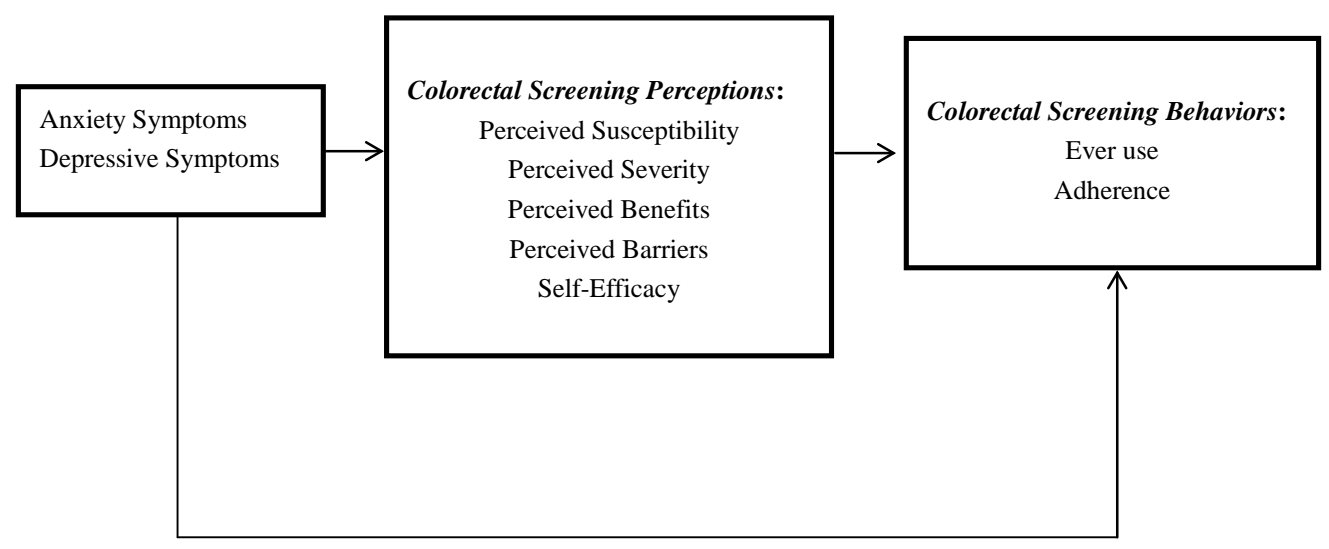

Figure 1. Theoretical framework for anxiety and depressive symptoms as predictors of colorectal cancer screening perceptions and behaviors.

Board at Eastern Virginia Medical School approved study procedures with a waiver of informed consent.

\subsection{Survey Instrument}

The anonymous self-administered questionnaire consisted of approximately 88 items, covering socio-demographic, lifestyle and health factors, symptoms of anxiety and depression as well as CRC screening perceptions and behaviors, with an estimated 20-minute time for completion. Questionnaire items were adapted from large surveillance systems such as the BRFSS and the NHIS as well as previously validated scales. Since fewer than $2 \%$ of primary care patients were non-English speakers, the questionnaire was not translated to other languages.

\subsection{Measures}

\subsubsection{Colorectal Cancer Screening Behaviors}

CRC screening ever use was measured with a set of questions that assessed whether or not a CRC screening test, including FOBT, sigmoidoscopy and colonoscopy, has been used in the past. Three "yes" or "no" questions aimed at assessing CRC screening ever use were phrased as follows: "Have you ever had a sigmoidoscopy?", "Have you ever had a colonoscopy to screen for colorectal polyps?” and "Have you ever had a FOBT?”. For patients who answered affirmatively, three follow-up questions were asked to evaluate the time duration since the last CRC testing: "When was the last time that you had a sigmoidoscopy?", "When was the last time that you had a colonoscopy to screen for colorectal polyps?", "When was the last time that you had a FOBT?”, with the following possible answers: “< 1 year ago", "1 - 4.9 years ago", "5 - 9.9 years ago", " $10+$ years ago" and "Don’t know". Accordingly, a patient identified as being adherent to CRC screening guidelines if he or she had undergone FOBT in the past 12 months, sigmoidoscopy in the past 5 years or colonoscopy in the past 10 years. Due to their limited use in the general population, use of barium enema or other CRC screening methods were not evaluated in the self-administered questionnaire. Questions that assess CRC screening behaviors were adapted from the existing literature, including studies that were based on the BRFSS and the NHIS data.

\subsubsection{Colorectal Cancer Screening Perceptions}

A series of 5-point Likert-type questions were adapted from existing studies to measure the various HBM constructs, namely, "perceived susceptibility" (1 item) [23, 46], "perceived severity" (3 items) [47], "perceived benefits" (12 items) [26,48,49], "perceived barriers" (15 items) [50] and "self-efficacy" (3 items) [26]. Perceived susceptibility was measured by asking the following statement: "Compared to other people of my age, my chances of having colorectal cancer in the future are ...”. The following statements were used to measure perceived severity: "Having colorectal cancer seriously disrupts health and comfort", "Colorectal cancer greatly influences a person emotionally" and "The health consequences of developing colorectal cancer are very severe". Self-efficacy was measured using three inter-related questions, namely, "How confident are you that you would be able to go for a sigmoidoscopy?", "How confident are you that you would be able to go for a screening colonoscopy?" and "How confident are you that you would be able to go for a FOBT testing?” The 12-item scale used to measure perceived benefits and the 15-item scale used to measure perceived barriers are presented in Table 1. For items pertaining to "perceived severity", "perceived benefits" and "perceived barriers", responses were coded as follows: 1 = "Strongly disagree", 2 = "Disagree", 3 = "Neutral", 4 = "Agree" and 5 = "Strongly Agree". Moreover, the "perceived susceptibility" item was designated as $(1=$ "Much less than other people of my age" $. .5=$ "Much more than other people of my age"), whereas the "self-efficacy" items were designated 
Table 1. Perceived benefits and barriers for colorectal cancer screening scale items* .

\section{Perceived Benefits:}

1. I would feel better about myself if I had colorectal screening.

2. I am confident that I could have colorectal cancer screening on a regular schedule.

3. Having regular colorectal cancer screening would give me a feeling of control over my health.

4. Colorectal screenings are now very routine tests.

5. My family would benefit if I had colorectal screenings.

6. Colorectal screening could find growths that are not yet cancer but might develop into cancer.

7. Colorectal screening is part of good overall health care.

8. Having regular colorectal screening would give me peace of mind about my health.

9. Colorectal cancer screening is safe.

10. I would be more likely to have colorectal screening if my doctor told me how important it was.

11. Colorectal screening is a useful procedure for people my age.

12. Colorectal screening would help me to live a long life.

\section{Perceived Barriers:}

1. If my doctor didn't mention colorectal screening, I probably would not need it.

2. Colorectal screening has a high risk of leading to unnecessary surgery.

3. If my doctor gave me a thorough examination, then I would not need any special colorectal screening.

4. I would probably not go for colorectal screening unless I had a specific symptom.

5. Once you have had a couple of colorectal screenings in a row that show no problems, you don't need any more.

6. I would probably not have colorectal screening if it involved enemas or laxatives to clear out my colon.

7. Having colorectal screening would be very embarrassing.

8. If colorectal screening found something, whatever the problem was, it would be too far along to do something about it.

9. It would be inconvenient to have colorectal screening at this time.

10. If there were any chance that colorectal screening is not safe, I would not want to have it.

11. The risk of harm from colorectal screening is really quite high.

12. If I maintained a healthy lifestyle, I would lower my risk of getting colorectal cancer and probably would not need to have colorectal screenings.

13. I cannot afford to have colorectal screening tests.

14. I would probably not have colorectal screening if a family member or friend said that it was painful.

15. Colorectal screening would interfere too much with other things I have to do.

"Item responses were coded as 1 = "Strongly disagree", 2 = "Disagree”, 3 = "Neutral”, 4 = "Agree” and 5 = "Strongly Agree”.

as $(1=$ "Not at all confident" $\cdots 5=$ "Extremely confident”). Using the study sample, internal consistenty reliability was estimated using Cronbach's alpha for each CRC screening perception that was measured using at least two items, namely "perceived severity" (alpha = 0.77), "self-efficacy" (alpha $=0.84)$, "perceived benefits" (alpha $=0.96)$ and "perceived barriers" (alpha $=0.94$ ). Each CRC screening perception was analyzed as a continuous variable by computing a total score for each scale, as needed. A higher total score is considered to indicate more self-efficacy, perceived susceptibility, severity, benefits or barriers.

\subsubsection{Symptoms of Anxiety and Depression}

The Hospital Anxiety and Depression (HAD) scale is a widely used and validated self-administered 14-item scale that includes two sub-scales. The first sub-scale includes seven Likert-type items that describe symptoms 
of anxiety: "I feel tense or wound up", "I still enjoy the things I used to enjoy", "I get a sort of frightening feeling as if something awful is about to happen", "I can laugh and see the funny side of things", "Worrying thoughts go through my mind", "I feel cheerful", "I can sit at ease and feel relaxed". The second sub-scale includes seven Likert-type items that describe symptoms of depression: "I feel as if I am slowed down", "I get a sort of frightened feeling like 'butterflies' in the stomach", "I have lost interest in my appearance", "I feel restless as if I have to be on the move", "I look forward with enjoyment to things", "I get sudden feelings of panic" and "I can enjoy a good book or radio or television program". Negative item responses are coded to range between 0 and 3, whereas positive item responses are reversescored. Thus, for each sub-scale, the total score can range between 0 and 21. An important strength of the HAD scale is that it does not cover somatic symptoms. Anxiety and depression sub-scales can be scored as follows: 1) 0 - 7 (None); 2) 8 - 10 (Mild); 3) 11 - 14 (Moderate); 4) 15 - 21 (Clinical) [51]. Due to sample size limitations, we dichotomized the anxiety and depression sub-scales as: 1 ) $<11$ (None/Mild); 2) $\geq 11$ (Moderate/ Clinical).

\subsubsection{Socio-Demographic Characteristics}

The following socio-demographic characteristics were identified as a-priori confounders for the hypothesized relationships: 1) age; 2) gender; 3) race and ethnicity; 4) marital status; 5) education; 6) Smoking history; 7) body mass index (BMI) and 8) Self-rated health. BMI was defined as [weight $(\mathrm{kg}) /(\text { height }(\mathrm{m}))^{2}$ ]. Also, self-rated health was measured using one questionnaire item phrased as follows: "When compared to people of the same age as you are now, would you describe your health as being excellent, very good, good, fair or poor?", with "Excellent”, "Very good", "Good”, "Fair" or "Poor" as potential responses.

\subsection{Statistical Analyses}

Descriptive, bivariate and multivariate analyses were performed using SAS (version 9.3) software. Cronbach's alpha was used to assess internal consistency reliability for scales pertaining to HBM constructs. Descriptive analyses involved frequencies and percentages for categorical variables and mean \pm standard deviation for numerical variables. Pearson's Chi-square, Wilcoxon's rank-sum and Kruskal-Wallis tests were applied to examine bivariate associations, as appropriate. Logistic and linear regression models were constructed to examine the hypothesized relationships, before and after adjustment for confounders. Two-sided statistical significance was determined at an alpha level of 0.05 [52]. Where needed, family-wise Bonferroni correction was applied to adjust for multiple testing.

\section{RESULTS}

A total of 143 English-speaking older adults, 50 to 79 years of age, completed the questionnaire. Nearly $38 \%$ of the study sample consisted of individuals 65 years or older, with a mean ( \pm standard deviation) age of $62.4( \pm$ 8.9) years; $55 \%$ were female and most of them were either of Black (53\%) or White (46\%) race. Whereas $85 \%$ had more than a high school level of education, 58\% were unmarried. In addition, 93\% were overweight or obese, 38\% had never smoked and 25\% rated their health as fair or poor.

The prevalence rates of moderate to clinical anxiety and depressive symptoms were $47 \%$ and $42 \%$, respectively, with a mean ( \pm standard deviation) anxiety score of $10.4( \pm 2.0)$ and a mean ( \pm standard deviation) depression score of 10.2 ( \pm 1.9$)$. No significant differences in anxiety or depressive symptoms were observed according to most of the socio-demographic, lifestyle and health characteristics examined. One exception was smoking status which was found to be significantly associated with depressive symptoms but not anxiety symptoms (Table 2).

Approximately $94 \%$ of the study sample had used any CRC screening test, whereas $89 \%$ were adherent to CRC screening recommendations. The most popular modality for CRC screening was "colonoscopy" (ever use: 81\%; adherence: 76\%) followed by "FOBT" (ever use: 59\%; adherence: 16\%) and "sigmoidoscopy" (ever use: 29\%; adherence: 13\%). Table 3 presents CRC screening perceptions and behaviors by socio-demographic, lifestyle and health characteristics of the study sample. Racial disparities were noted whereby minorities were less likely to have ever used or to be adherent to CRC testing. Minorities also perceived more barriers and less self-efficacy as compared to White patients. An inverted U-shaped relationship was observed between perceived severity and BMI. Also, current smokers were less likely than never smokers or ex-smokers to have ever used CRC screening. Finally, individuals with fair or poor self-rated health had a lower self-efficacy and perceived fewer benefits and greater barriers to CRC screening. Few of these associations remained significant after Bonferroni correction.

Table 4 presents the unadjusted and adjusted odds ratios (OR) and their 95\% confidence intervals (CI) for the relationships between anxiety/depression symptoms and CRC screening behaviors. Taking all CRC screening modalities into account, neither anxiety nor depressive symptoms were shown to be associated with ever use or adherence to CRC testing.

Linear regression analyses for anxiety and depressive symptoms as predictors of CRC screening perceptions 
Table 2. Anxiety and depression characteristics by socio-demographic factors in study sample.

\begin{tabular}{|c|c|c|c|c|c|c|}
\hline & \multicolumn{3}{|c|}{ Anxiety Symptoms } & \multicolumn{3}{|c|}{ Depressive Symptoms } \\
\hline & Mean \pm SD & $<11$ & $\geq 11$ & Mean \pm SD & $<11$ & $\geq 11$ \\
\hline Overall & $10.4 \pm 2.0$ & $74(100)$ & $65(100)$ & $10.2 \pm 1.9$ & $80(100)$ & $58(100)$ \\
\hline Gender: & $\mathrm{P}=0.17$ & \multicolumn{2}{|c|}{$\mathrm{P}=0.29$} & $\mathrm{P}=0.50$ & \multicolumn{2}{|c|}{$\mathrm{P}=0.38$} \\
\hline Male & $10.6 \pm 2.1$ & 31 (41.8) & $33(50.8)$ & $10.3 \pm 1.8$ & $34(42.5)$ & $29(50.0)$ \\
\hline Female & $10.2 \pm 1.9$ & $43(58.1)$ & $32(49.2)$ & $10.1 \pm 2.0$ & 46 (57.5) & $29(50.0)$ \\
\hline Age (years): & $P=0.063$ & \multicolumn{2}{|c|}{$P=0.16$} & $\mathrm{P}=0.44$ & \multicolumn{2}{|c|}{$\mathrm{P}=0.43$} \\
\hline $50-64$ & $10.1 \pm 1.9$ & $43(67.2)$ & $33(55.0)$ & $10.2 \pm 1.7$ & 43 (58.9) & $33(66.0)$ \\
\hline$\geq 65$ & $10.8 \pm 2.2$ & $21(32.8)$ & $27(45.0)$ & $10.0 \pm 1.8$ & $30(41.1)$ & $17(34.0)$ \\
\hline Race: & $\mathrm{P}=0.28$ & \multicolumn{2}{|c|}{$\mathrm{P}=0.13$} & $\mathrm{P}=0.46$ & \multicolumn{2}{|c|}{$\mathrm{P}=0.94$} \\
\hline White & $10.2 \pm 1.8$ & 38 (52.8) & $24(40.7)$ & $10.0 \pm 1.6$ & 35 (46.1) & $26(48.2)$ \\
\hline Black & $10.5 \pm 2.2$ & $32(44.4)$ & 35 (59.3) & $10.3 \pm 1.8$ & $40(52.6)$ & $27(50.0)$ \\
\hline Other & $8.5 \pm 2.1$ & $2(2.8)$ & $0(0.0)$ & $12.5 \pm 3.5$ & $1(1.3)$ & $1(1.9)$ \\
\hline Marital status: & $\mathrm{P}=0.47$ & \multicolumn{2}{|c|}{$\mathrm{P}=0.14$} & $\mathrm{P}=0.21$ & \multicolumn{2}{|c|}{$\mathrm{P}=0.11$} \\
\hline Married & $10.2 \pm 1.9$ & 35 (47.3) & $22(34.9)$ & $10.3 \pm 1.8$ & $28(35.4)$ & $28(49.1)$ \\
\hline Not married & $10.5 \pm 2.1$ & $39(52.7)$ & $41(65.1)$ & $10.1 \pm 2.0$ & $51(64.6)$ & $29(50.9)$ \\
\hline Education: & $\mathrm{P}=0.82$ & \multicolumn{2}{|c|}{$\mathrm{P}=0.39$} & $\mathrm{P}=0.74$ & \multicolumn{2}{|c|}{$\mathrm{P}=0.52$} \\
\hline Less than HS & $9.4 \pm 0.4$ & $2(2.7)$ & $0(0.0)$ & $9.7 \pm 2.3$ & $1(1.3)$ & $2(3.5)$ \\
\hline HS & $10.6 \pm 2.5$ & $9(12.3)$ & $9(13.9)$ & $10.6 \pm 2.4$ & $9(11.4)$ & $9(15.5)$ \\
\hline More than HS & $10.4 \pm 1.9$ & $62(84.9)$ & $56(86.2)$ & $10.2 \pm 1.8$ & $69(87.3)$ & $47(81.0)$ \\
\hline Body mass index $\left(\mathrm{kg} / \mathrm{m}^{2}\right)$ : & $\mathrm{P}=0.23$ & \multicolumn{2}{|c|}{$\mathrm{P}=0.34$} & $\mathrm{P}=0.66$ & \multicolumn{2}{|c|}{$\mathrm{P}=0.39$} \\
\hline$<25$ & $11.4 \pm 1.2$ & $2(3.5)$ & $5(9.4)$ & $10.9 \pm 2.4$ & $3(4.6)$ & $5(11.1)$ \\
\hline $25-29.9$ & $10.4 \pm 2.0$ & 23 (39.7) & $23(43.4)$ & $10.0 \pm 1.7$ & $29(43.9)$ & $17(37.8)$ \\
\hline$\geq 30$ & $10.2 \pm 2.0$ & 33 (56.9) & $25(27.2)$ & $10.1 \pm 1.8$ & $34(51.5)$ & $23(51.1)$ \\
\hline Smoking status: & $\mathrm{P}=0.66$ & \multicolumn{2}{|c|}{$\mathrm{P}=0.67$} & $\mathrm{P}=0.047$ & \multicolumn{2}{|c|}{$\mathrm{P}=0.037$} \\
\hline Never smoker & $10.7 \pm 2.1$ & $29(40.9)$ & $22(35.5)$ & $10.3 \pm 1.9$ & 27 (35.5) & $23(41.1)$ \\
\hline Ex-smoker & $10.3 \pm 1.8$ & $30(42.3)$ & $26(41.9)$ & $10.4 \pm 1.7$ & 27 (35.5) & $27(48.2)$ \\
\hline Current smoker & $10.0 \pm 2.0$ & $12(16.9)$ & $14(22.6)$ & $9.4 \pm 1.5$ & $22(28.9)$ & $6(10.7)$ \\
\hline Self-rated Health: & $\mathrm{P}=0.41$ & \multicolumn{2}{|c|}{$\mathrm{P}=0.40$} & $\mathrm{P}=0.45$ & \multicolumn{2}{|c|}{$P=0.16$} \\
\hline Excellent/Very Good/Good & $10.3 \pm 1.8$ & $57(78.1)$ & 46 (71.9) & $10.2 \pm 1.9$ & $57(72.2)$ & $47(82.5)$ \\
\hline Fair/Poor & $10.8 \pm 2.6$ & $16(21.9)$ & 18 (28.1) & $10.1 \pm 2.1$ & 22 (27.8) & $10(17.5)$ \\
\hline
\end{tabular}

are presented in Table 5. Clearly, perceived benefits and barriers were the only HBM constructs that were significantly associated with symptoms of anxiety, and none of the HBM constructs were significantly associated with symptoms of depression. Perceived benefits were found to be negatively associated with anxiety symptoms in the unadjusted but not in the adjusted regression model. By contrast, perceived barriers were found to be positively associated with anxiety symptoms after adjustment for confounders.

\section{DISCUSSION}

This cross-sectional study examined whether symptoms of anxiety and depression were associated with perceptions and behaviors pertaining to CRC screening. 
Table 3. Colorectal cancer screening perceptions and behaviors by socio-demographic factors in study sample.

\begin{tabular}{|c|c|c|c|c|c|c|c|}
\hline & \multicolumn{2}{|c|}{ CRC Behaviors } & \multicolumn{5}{|c|}{ CRC Perceptions } \\
\hline & Ever Use & Adherence & Susceptibility & Severity & Benefits & Barriers & Self-Efficacy \\
\hline Overall & 93.7 & 88.8 & $2.8 \pm 1.2$ & $12.0 \pm 2.5$ & $48.1 \pm 8.9$ & $35.2 \pm 10.5$ & $11.8 \pm 3.6$ \\
\hline Gender: & $\mathrm{P}=0.21$ & $\mathrm{P}=0.16$ & $\mathrm{P}=0.91$ & $\mathrm{P}=0.42$ & $P=0.32$ & $\mathrm{P}=0.062$ & $\mathrm{P}=0.078$ \\
\hline Male & 96.6 & 93.1 & $2.8 \pm 1.2$ & $12.3 \pm 2.3$ & $49.1 \pm 8.1$ & $33.4 \pm 10.8$ & $12.2 \pm 3.7$ \\
\hline Female & 91.2 & 85.1 & $2.8 \pm 1.2$ & $11.9 \pm 2.6$ & $47.3 \pm 9.5$ & $36.7 \pm 10.1$ & $11.5 \pm 3.5$ \\
\hline Age (years): & $\mathrm{P}=0.58$ & $\mathrm{P}=0.71$ & $\mathrm{P}=0.32$ & $\mathrm{P}=0.64$ & $\mathrm{P}=0.46$ & $\mathrm{P}=0.47$ & $\mathrm{P}=0.93$ \\
\hline $50-64$ & 92.8 & 88.2 & $2.8 \pm 1.2$ & $11.9 \pm 2.4$ & $48.2 \pm 8.4$ & $35.2 \pm 9.8$ & $11.8 \pm 3.7$ \\
\hline$\geq 65$ & 95.4 & 90.5 & $2.6 \pm 1.2$ & $12.1 \pm 2.7$ & $48.8 \pm 9.2$ & $34.3 \pm 11.5$ & $11.8 \pm 3.7$ \\
\hline Race: & $\mathrm{P}=0.0005^{* \dagger}$ & $\mathrm{P}=0.0082^{*}$ & $\mathrm{P}=0.52$ & $\mathrm{P}=0.28$ & $\mathrm{P}=0.53$ & $\mathrm{P}=0.0021^{* \dagger}$ & $\mathrm{P}=0.031^{*}$ \\
\hline White & 96.7 & 93.2 & $2.7 \pm 1.2$ & $12.4 \pm 2.4$ & $48.6 \pm 9.3$ & $32.3 \pm 10.1$ & $12.4 \pm 3.6$ \\
\hline Black & 91.4 & 86.2 & $2.9 \pm 1.2$ & $11.9 \pm 2.5$ & $48.4 \pm 7.8$ & $36.7 \pm 9.6$ & $6.5 \pm 4.9$ \\
\hline Other & 0.0 & 0.0 & $2.0 \pm 1.4$ & $10.5 \pm 2.1$ & $42.0 \pm 8.5$ & $57.5 \pm 3.5$ & $6.5 \pm 4.9$ \\
\hline Marital status: & $\mathrm{P}=0.11$ & $P=0.33$ & $P=0.10$ & $P=0.43$ & $P=0.46$ & $P=0.27$ & $P=0.48$ \\
\hline Married & 98.2 & 92.5 & $2.9 \pm 1.2$ & $12.3 \pm 2.4$ & $49.0 \pm 8.9$ & $33.9 \pm 11.0$ & $12.2 \pm 3.4$ \\
\hline Not married & 91.4 & 86.9 & $2.6 \pm 1.2$ & $11.9 \pm 2.5$ & $47.9 \pm 8.8$ & $35.8 \pm 10.2$ & $11.7 \pm 3.8$ \\
\hline Education: & $\mathrm{P}=0.97$ & $\mathrm{P}=0.13$ & $\mathrm{P}=0.28$ & $\mathrm{P}=0.29$ & $\mathrm{P}=0.21$ & $\mathrm{P}=0.10$ & $P=0.063$ \\
\hline Less than HS & 100 & 100 & $3.7 \pm 1.2$ & $11.3 \pm 3.2$ & $40.7 \pm 5.0$ & $39.0 \pm 8.0$ & $12.3 \pm 3.0$ \\
\hline HS & 93.3 & 73.3 & $2.9 \pm 0.8$ & $11.2 \pm 2.7$ & $46.3 \pm 10.4$ & $40.5 \pm 11.7$ & $10.1 \pm 3.9$ \\
\hline More than HS & 93.6 & 90.7 & $2.7 \pm 1.3$ & $12.2 \pm 2.5$ & $48.5 \pm 8.7$ & $34.1 \pm 10.2$ & $12.0 \pm 3.5$ \\
\hline Body mass index $\left(\mathrm{kg} / \mathrm{m}^{2}\right)$ : & $\mathrm{P}=0.10$ & $\mathrm{P}=0.38$ & $\mathrm{P}=0.25$ & $\mathrm{P}=0.028$ & $\mathrm{P}=0.39$ & $\mathrm{P}=0.18$ & $\mathrm{P}=0.66$ \\
\hline$<25$ & 66.7 & 66.7 & $3.1 \pm 0.8$ & $10.6 \pm 1.4$ & $44.3 \pm 8.3$ & $42.4 \pm 14.7$ & $12.8 \pm 2.8$ \\
\hline $25-29.9$ & 90.5 & 85.4 & $2.6 \pm 1.2$ & $12.7 \pm 1.9$ & $48.6 \pm 8.5$ & $33.3 \pm 9.7$ & $11.9 \pm 3.6$ \\
\hline$\geq 30$ & 96.4 & 90.7 & $2.9 \pm 1.2$ & $11.7 \pm 2.8$ & $48.0 \pm 9.2$ & $36.4 \pm 9.8$ & $11.6 \pm 3.7$ \\
\hline Smoking status: & $\mathrm{P}=0.042^{*}$ & $\mathrm{P}=0.14$ & $\mathrm{P}=0.85$ & $\mathrm{P}=0.88$ & $\mathrm{P}=0.79$ & $\mathrm{P}=0.22$ & $\mathrm{P}=0.53$ \\
\hline Never smoker & 93.3 & 88.9 & $2.8 \pm 1.2$ & $12.2 \pm 2.3$ & $48.4 \pm 8.5$ & $36.2 \pm 11.4$ & $12.1 \pm 3.6$ \\
\hline Ex-smoker & 98.1 & 92.6 & $2.7 \pm 1.3$ & $11.9 \pm 2.8$ & $47.4 \pm 9.2$ & $33.3 \pm 9.9$ & $11.5 \pm 3.7$ \\
\hline Current smoker & 82.6 & 76.2 & $2.7 \pm 1.1$ & $11.9 \pm 2.4$ & $48.8 \pm 9.6$ & $36.9 \pm 9.9$ & $11.7 \pm 3.6$ \\
\hline Self-rated Health: & $P=0.44$ & $P=0.36$ & $\mathrm{P}=0.095$ & $P=0.60$ & $\mathrm{P}=0.018^{*}$ & $\mathrm{P}=0.0078^{*}$ & $\mathrm{P}=0.015^{*}$ \\
\hline Excellent/Very Good/Good & 92.7 & 87.2 & $2.6 \pm 1.2$ & $12.1 \pm 2.5$ & $49.2 \pm 8.5$ & $33.8 \pm 10.6$ & $12.3 \pm 3.3$ \\
\hline Fair/Poor & 96.7 & 93.3 & $3.0 \pm 1.3$ & $11.8 \pm 2.6$ & $44.7 \pm 9.4$ & $39.4 \pm 9.6$ & $10.3 \pm 4.2$ \\
\hline
\end{tabular}

*Significant at alpha $=0.05$, before family-wise Bonferroni adjustment; ${ }^{\dagger}$ Significant at alpha $=0.05$, after family-wise Bonferroni adjustment.

In this primary care population, although the vast majority had undergone or were adherent to screening recommendations using FOBT, sigmoidoscopy and/or colonoscopy, fewer were adherent with CRC screening recommendations. However, the preferred method of CRC screening appears to be colonoscopy followed by FOBT and sigmoidoscopy. Our study results suggest that symp- toms of anxiety and depression may not be related to CRC screening behaviors, including ever use or adherence to guidelines. Irrespective of whether or not a patient had previously undergone CRC screening or is adherent to CRC screening recommendations, symptoms of anxiety and depression appear to influence CRC perceptions, which in turn could affect future use of CRC test- 
Table 4. Logistic Regression models for Anxiety and Depressive Symptoms as predictors of Colorectal Cancer Screening Behaviors.

\begin{tabular}{|c|c|c|c|c|}
\hline & \multicolumn{4}{|c|}{ CRC Behaviors } \\
\hline & \multicolumn{2}{|c|}{ Ever Use } & \multicolumn{2}{|c|}{ Adherence } \\
\hline & cOR $(95 \%$ CI) & aOR (95\% CI) & cOR (95\% CI) & aOR (95\% CI) \\
\hline \multicolumn{5}{|c|}{ Anxiety Symptoms: } \\
\hline Continuous & $1.06(0.73-1.53)$ & $1.22(0.65-2.29)$ & $1.15(0.86-1.52)$ & $1.19(0.76-1.89)$ \\
\hline$\geq 11$ & $0.63(0.14-2.95)$ & $0.52(0.04-6.90)$ & $1.01(0.32-3.19)$ & $0.67(0.12-3.65)$ \\
\hline \multicolumn{5}{|c|}{ Depressive Symptoms: } \\
\hline Continuous & $1.08(0.72-1.62)$ & $1.02(0.51-2.06)$ & $1.08(0.79-1.49)$ & $1.14(0.68-1.91)$ \\
\hline$\geq 11$ & $5.78(0.69-48.48)$ & $4.71(0.34-64.63)$ & $2.11(0.62-7.16)$ & $2.88(0.48-17.15)$ \\
\hline
\end{tabular}

Table 5. Linear Regression Models for Anxiety and Depressive Symptoms as predictors of Colorectal Cancer Screening Perceptions.

\begin{tabular}{cc}
\hline \multicolumn{2}{c}{ CRC Perceptions } \\
\hline Unadjusted & Multivariate \\
$\beta(\mathrm{P})$ & $\beta(\mathrm{P})$
\end{tabular}

Perceived Susceptibility:

Anxiety Symptoms:

$\begin{array}{ccc}\text { Continuous } & -0.015(0.77) & -0.027(0.71) \\ \geq 11 & -0.20(0.33) & -0.077(0.79)\end{array}$

Depressive Symptoms:

$\begin{array}{ccc}\text { Continuous } & 0.0074(0.89) & -0.046(0.56) \\ \geq 11 & 0.26(0.22) & 0.13(0.63)\end{array}$

Perceived Severity:

Anxiety Symptoms:

$\begin{array}{ccc}\text { Continuous } & -0.029(0.78) & 0.076(0.58) \\ \geq 11 & -0.63(0.13) & -0.023(0.97)\end{array}$

Depressive Symptoms:

$\begin{array}{ccc}\text { Continuous } & -0.084(0.45) & -0.073(0.63) \\ \geq 11 & -0.14(0.74) & -0.52(0.33)\end{array}$

Perceived Benefits:

Anxiety Symptoms:

$$
\begin{array}{ccc}
\text { Continuous } & -0.76(0.038)^{*} & -0.56(0.22) \\
\geq 11 & -3.03(0.042)^{*} & -3.25(0.075)
\end{array}
$$

Depressive Symptoms:

$\begin{array}{cll}\text { Continuous } & 0.43(0.27) & 0.49(0.32) \\ \geq 11 & 0.58(0.71) & 0.25(0.88)\end{array}$

Perceived Barriers:

\section{Continued}

Anxiety Symptoms:

$\begin{array}{lll}\text { Continuous } & 0.78(0.075) & 1.24(0.028)^{*} \\ \geq 11 & 3.11(0.082) & 6.39(0.0042)^{*} \\ \text { essive Symptoms: } & & \\ \text { Continuous } & -0.014(0.98) & -0.53(0.39) \\ \geq 11 & -1.49(0.42) & -2.13(0.33)\end{array}$

Perceived Self-Efficacy:

Anxiety Symptoms:

$\begin{array}{ccc}\text { Continuous } & -0.10(0.51) & -0.18(0.38) \\ \geq 11 & -0.60(0.33) & -1.26(0.13)\end{array}$

Depressive Symptoms:

$\begin{array}{ccc}\text { Continuous } & 0.093(0.56) & 0.10(0.66) \\ \geq 11 & 0.14(0.82) & -0.28(0.72)\end{array}$

*Significant at alpha $=0.05$.

ing. Our results suggest that of all the CRC screening perceptions evaluated, "perceived benefits" and "perceived barriers" were the only significant correlates of anxiety/depression symptoms.

To our knowledge, this study is the first to have examined symptoms of anxiety and depression as predictors of CRC perceptions, although it is not the first to have examined these symptoms in relation to CRC behaviors. These findings should, nevertheless, be interpreted with caution and in light of several limitations. First, the cross-sectional nature of the study design precludes the evaluation of temporal relationships among exposure and outcome variables of interest. Second, a convenience sampling procedure was applied which increases the likelihood of selection bias. Third, the survey was conducted at one family medicine clinic limiting generalizability to other primary care settings. Of note, certain 
characteristics of the selected sample including the prevalence of CRC use and the propensity to use colonoscopy as the preferred method suggest that it may not be typical of age-eligible individuals in the U.S.

The vast majority of primary care patients in this clinical setting had at least one form of health insurance coverage, which may explain the greater usage of CRC screening tests compared to the general population of the United States. BRFSS (1991-2004) trends suggest that each year less than $60 \%$ of men and women 50 years of age or older have used CRC screening services based on established guidelines [1,53]. For instance, the 1999 BRFSS indicated that only $40 \%$ of age-eligible older adults ever received a home-administered FOBT and 44\% had undergone either sigmoidoscopy or colonoscopy. In addition, 21\% received FOBT in the preceding year and $34 \%$ had a LE within the preceding 5 years [54]. Similarly, the 2004 BRFSS indicated that $57.3 \%$ of older adults had an FOBT within one year or a LE within 10 years preceding the survey [55]. Furthermore, the preferred use of colonoscopy by the selected patients may be the result of a general trend in physician recommendations at this particular family medicine practice.

Our research team has previously conducted a systematic review of the literature whereby predictors of CRC screening behaviors among average-risk older adults ( $\geq 50$ years) in the United States were evaluated. A PUBMED (1996-2006) search indicated that older age, male gender, marriage, higher education, higher income, White race, non-Hispanic ethnicity, smoking history, presence of chronic diseases, family history of CRC, usual source of care, physician recommendation, utilization of other preventive health services, and health insurance coverage as key predictors of CRC testing [27].

The present study is one of few to have examined mental health problems in relation to cancer screening behaviors [28-45]. The evidence from previous studies remains inconclusive. Recently, Yee [44] evaluated the association of mental illness with receipt and adherence to breast, cervical and CRC screening among female Veterans, 50 - 65 years who obtained care in New Mexico between 2004 and 2006. Women with a mental health diagnosis were less likely to adhere to recommended breast cancer screening but were as likely as those without a mental health diagnosis to receive any breast, cervical and colon cancer screening [44]. Another study by Xiong [43] examined the use of breast, cervical, colorectal and prostate cancer screening services by persons with serious mental illness enrolled in the Sacramento County Mental Health clinics. Among persons with serious mental illness, lifetime screening of cervical cancer was higher than for breast, prostate, and colorectal cancers [43]. Receipt of routine, timely cancer screening was low, especially for CRC [43]. A study by Friedman
[38] examined predictors of breast and CRC screening among 196 low-income women being treated for psychiatric illnesses. The main outcome measures were breast self-examination (BSE), clinical breast examination (CBE) mammography, digital rectal examination (DRE) and fecal occult blood test (FOBT) [38]. Multivariate analyses suggested that physician recommendation of screening was the strongest predictor of having obtained a mammogram, CBE, DRE or FOBT in the preceding year, and physician recommendation and selfconfidence in performing BSE were the strongest predictors of monthly BSE [38]. The authors concluded that because psychiatrists frequently treat these patients on a regular basis, they are in a unique position to encourage cancer screening and to monitor adherence to recommendations [38].

The finding that depressive symptoms may be associated with use of CRC screening has been observed elsewhere, but remains controversial. A study by Peytremann [42] investigated the association between depressive symptoms and use of healthcare and preventive services. Data were analyzed on a total of 13,580 noninstitutionalized individuals aged $\geq 50$ years participating in the Survey of Health, Ageing, and Retirement in Europe [42]. Depressive symptoms were associated with significantly greater use of all healthcare domains but not preventive services, with the exception of CRC screening [42]. Similar trends were found for each country of residence and for both genders [42]. In contrast, Myong [40] used a sample of 8042 research participants $\geq 50$ years, who participated in the KNHANES IV and found that compliance with CRC recommendation was associated with age, marital status, urban-dwelling, education, household income, private health insurance, smoking, self-reported depression (inverse relationship) and number of chronic diseases. Aggrawal [37] relied on the Women's Health Initiative Observational Study whereby 93,676 women were followed on average for 7.6 years and depressive symptoms were measured at baseline and at 3 years using a 6-item sub-scale from the CES-D [37]. A cancer screening rate was calculated and expressed as a proportion of the years that women were current with recommended cancer screening over the number of follow-up visits. Whereas breast cancer screening rate was slightly lower among women who reported depressive symptoms at baseline than among those who did not, depressive symptoms were not a predictor for CRC screening [37].

Few studies have specifically examined mental health problems in relation to CRC screening. A study by Kodl [39] examined the association between mental health and CRC screening, before and after controlling for demographics, comorbidities, and outpatient visit frequency, using data on 855 Veterans Administration patients. 
Medical records data were used to assess CRC screening rates and mental health status (number of diagnoses, presence of depression, anxiety, posttraumatic stress disorder, substance or psychotic disorders) [39]. Veterans with mental health diagnoses were significantly less likely to be screened for CRC than their counterparts with no mental health diagnosis and an equal number of outpatient visits [39]. O’Donnell and colleagues examined the impact of women's attitudes and health beliefs regarding breast and CRC screening practices [41]. A total of 905 women, 50 to 80 years of age, were recruited from 63 randomly selected physician offices and the HBM was used to evaluate potential predictor variables associated with patients' breast and CRC screening practices [41]. Results indicated that women were far more likely to obtain regular mammography screening than an FOBT [41]. Psychological distress had one of the strongest, negative associations with breast cancer and CRC screening, and was also a prevalent predictor for many of the variables examined [41].

\section{CONCLUSION}

In conclusion, evidence remains divided as to whether symptoms of anxiety and depression may influence the likelihood of using and being adherent to CRC screening guidelines. Our study found no significant association between CRC screening behaviors and symptoms of anxiety or depression, which implied that these associations may be eclipsed by more important predictors such as availability of health insurance. The study also implicates anxiety, but not depression, as a predictor of perceived benefits and barriers for CRC screening, although neither of these symptoms was related to CRC screening behaviors. Healthcare professionals need to be cognizant of mental health issues that may constitute a barrier to use and adherence to cancer screening guidelines. Study findings suggest that anxiety may play a role in CRC screening perceptions, with implications for the future design, conduct and evaluation of behavioral interventions. Screening for anxiety and depression may be a useful tool for identifying patients who may or may not comply with physician recommendations pertaining to CRC screening. Large prospective cohort studies are needed to better understand the link between anxiety/ depression and cancer screening perceptions and behaviors.

\section{ACKNOWLEDGEMENTS}

This project was partly supported by the National Institute on Aging Intramural Research Program.

\section{REFERENCES}

[1] Matthews, B.A., Anderson, R.C. and Nattinger, A.B.
(2005) Colorectal cancer screening behavior and health insurance status (United States). Cancer Causes Control, 16, 735-742. http://dx.doi.org/10.1007/s10552-005-1228-z

[2] Janz, N.K., Wren, P.A., Schottenfeld, D. and Guire, K.E. (2003) Colorectal cancer screening attitudes and behavior: A population-based study. Preventive Medicine, 37, 627634. http://dx.doi.org/10.1016/j.ypmed.2003.09.016

[3] Hawk, E.T. and Levin, B. (2005) Colorectal cancer prevention. Journal of Clinical Oncology, 23, 378-391. http://dx.doi.org/10.1200/JCO.2005.08.097

[4] (1999) Harvard Report on Cancer Prevention: Prevention of colon cancer in the United States. Cancer Causes and Control, 10, 167-180. http://dx.doi.org/10.1023/A:1017117109568

[5] Huang, C.S., Lal, S.K. and Farraye, F.A. (2005) Colorectal cancer screening in average risk individuals. Cancer Causes Control, 16, 171-188. http://dx.doi.org/10.1007/s10552-004-4027-z

[6] Maciosek, M.V., Solberg, L.I., Coffield, A.B., Edwards, N.M. and Goodman, M.J. (2006) Colorectal cancer screening: Health impact and cost effectiveness. American Journal of Preventive Medicine, 31, 80-89.

http://dx.doi.org/10.1016/j.amepre.2006.03.009

[7] Diggs, J.C., Xu, F., Diaz, M., Cooper, G.S. and Koroukian, S.M. (2007) Failure to screen: predictors and burden of emergency colorectal cancer resection. American Journal of Managed Care, 13, 157-164.

[8] Christman, L.K., Abdulla, R., Jacobsen, P.B., Cantor, A.B., Mayhew, D.Y., Thompson, K.S., Krischer, J.P. and Roetzheim, R.G. (2004) Colorectal cancer screening among a sample of community health center attendees. Journal of Health Care for the Poor and Underserved, 15, 281-293. http://dx.doi.org/10.1353/hpu.2004.0021

[9] Etzioni, D.A., Yano, E.M., Rubenstein, L.V., Lee, M.L., Ko, C.Y., Brook, R.H., Parkerton, P.H. and Asch, S.M. (2006) Measuring the quality of colorectal cancer screening: the importance of follow-up. Diseases of the Colon and Rectum, 49, 1002-1010. http://dx.doi.org/10.1007/s10350-006-0533-2

[10] Lai, S.M., Zhang, K.B., Uhler, R.J., Harrison, J.N., Clutter, G.G. and Williams, M.A, (2006) Geographic variation in the incidence of colorectal cancer in the United States, 1998-2001. Cancer, 107, 1172-1180. http://dx.doi.org/10.1002/cncr.22014

[11] Collins, V., Meiser, B., Gaff, C., St John, D.J. and Halliday, J. (2005) Screening and preventive behaviors one year after predictive genetic testing for hereditary nonpolyposis colorectal carcinoma. Cancer, 104, 273-281. http://dx.doi.org/10.1002/cncr.21183

[12] Ko, C.W., Kreuter, W. and Baldwin, L.M. (2002) Effect of medicare coverage on use of invasive colorectal cancer screening tests. Archives of Internal Medicine, 162, 25812586. http://dx.doi.org/10.1001/archinte.162.22.2581

[13] Zimmerman, R.K., Tabbarah, M., Trauth, J., Nowalk, M.P. and Ricci, E.M. (2006) Predictors of lower endoscopy use among patients at three inner-city neighborhood health centers. Journal of Urban Health, 83, 221-230. http://dx.doi.org/10.1007/s11524-005-9028-z 
[14] Klabunde, C.N., Schenck, A.P. and Davis, W.W. (2006) Barriers to colorectal cancer screening among Medicare consumers. American Journal of Preventive Medicine, 30, 313-319. http://dx.doi.org/10.1016/j.amepre.2005.11.006

[15] Meissner, H.I., Breen, N., Klabunde, C.N. and Vernon, S.W. (2006) Patterns of colorectal cancer screening uptake among men and women in the United States. Cancer Epidemiology, Biomarkers \& Prevention, 15, 389-394. http://dx.doi.org/10.1158/1055-9965.EPI-05-0678

[16] Pollack, L.A., Blackman, D.K., Wilson, K.M., Seeff, L.C. and Nadel, M.R. (2006) Colorectal cancer test use among Hispanic and non-Hispanic U.S. populations. Preventing Chronic Disease, 3, A50.

[17] Shah, M., Zhu, K. and Potter, J. (2006) Hispanic acculturation and utilization of colorectal cancer screening in the United States. Cancer Detection and Prevention, 30, 306312. http://dx.doi.org/10.1016/j.cdp.2006.04.003

[18] Ahluwalia, I.B., Mack, K.A., Murphy, W., Mokdad, A.H. and Bales, V.S. (2003) State-specific prevalence of selected chronic disease-related characteristics-Behavioral risk factor surveillance system, 2001. MMWR Surveill Summ, 52, 1-80.

[19] Nelson, D.E., Bolen, J., Marcus, S., Wells, H.E. and Meissner, H. (2003) Cancer screening estimates for U.S. metropolitan areas. American Journal of Preventive Medicine, 24, 301-309.

http://dx.doi.org/10.1016/S0749-3797(03)00024-2

[20] Goodman, M.J., Ogdie, A., Kanamori, M.J., Canar, J. and O’Malley, A.S. (2006) Barriers and facilitators of colorectal cancer screening among Mid-Atlantic Latinos: Focus group findings. Ethnicity \& Disease, 16, 255-261.

[21] James, T.M., Greiner, K.A., Ellerbeck, E.F., Feng, C. and Ahluwalia, J.S. (2006) Disparities in colorectal cancer screening: A guideline-based analysis of adherence. Ethnicity \& Disease, 16, 228-233.

[22] Gilbert, A. and Kanarek, N. (2005) Colorectal cancer screening: Physician recommendation is influential advice to Marylanders. Preventive Medicine, 41, 367-379. http://dx.doi.org/10.1016/j.ypmed.2005.01.008

[23] Tessaro, I., Mangone, C., Parkar, I. and Pawar, V. (2006) Knowledge, barriers, and predictors of colorectal cancer screening in an Appalachian church population. Preventing Chronic Disease, 3, A123.

[24] Gorin, S.S. and Heck, J.E. (2005) Cancer screening among Latino subgroups in the United States. Preventive Medicine, 40, 515-526.

http://dx.doi.org/10.1016/j.ypmed.2004.09.031

[25] Greenwald, B. (2006) Promoting community awareness of the need for colorectal cancer screening: A pilot study. Cancer Nursing, 29, 134-141. http://dx.doi.org/10.1097/00002820-200603000-00009

[26] Menon, U., Belue, R., Sugg Skinner, C., Rothwell, B.E. and Champion, V. (2007) Perceptions of colon cancer screening by stage of screening test adoption. Cancer Nursing, 30, 178-185.

http://dx.doi.org/10.1097/01.NCC.0000270706.80037.05

[27] Beydoun, H.A. and Beydoun, M.A. (2008) Predictors of colorectal cancer screening behaviors among average-risk older adults in the United States. Cancer Causes Control, 19, 339-359.

http://dx.doi.org/10.1007/s10552-007-9100-y

[28] Linder, S.K., Swank, P.R., Vernon, S.W., Morgan, R.O., Mullen, P.D. and Volk, R.J. (2012) Is a prostate cancer screening anxiety measure invariant across two different samples of age-appropriate men? BMC Medical Informatics and Decision Making, 12, 52.

[29] Byrne, M.M., Weissfeld, J. and Roberts, M.S. (2008) Anxiety, fear of cancer, and perceived risk of cancer following lung cancer screening. Medical Decision Making: An International Journal of the Society for Medical Decision Making, 28, 917-925.

[30] Carlsson, S., Aus, G., Wessman, C. and Hugosson, J. (2007) Anxiety associated with prostate cancer screening with special reference to men with a positive screening test (elevated PSA)—Results from a prospective, population-based, randomised study. European Journal of Cancer, 43, 2109-2116. http://dx.doi.org/10.1016/j.ejca.2007.06.002

[31] Miles, A. and Wardle, J. (2006) Adverse psychological outcomes in colorectal cancer screening: Does health anxiety play a role? Behaviour Research and Therapy, 44, 1117-1127. http://dx.doi.org/10.1016/j.brat.2005.08.011

[32] Consedine, N.S., Magai, C., Krivoshekova, Y.S., Ryzewicz, L. and Neugut, A.I. (2004) Fear, anxiety, worry, and breast cancer screening behavior: A critical review. Cancer Epidemiology, Biomarkers \& Prevention: A Publication of the American Association for Cancer Research, Cosponsored by the American Society of Preventive Oncology, 13, 501-510.

[33] Hensley, M.L., Robson, M.E., Kauff, N.D., Korytowsky, B, Castiel, M., Ostroff, J., Hurley, K., Hann, L.E., Colon, J. and Spriggs, D. (2003) Pre- and postmenopausal highrisk women undergoing screening for ovarian cancer: Anxiety, risk perceptions, and quality of life. Gynecologic Oncology, 89, 440-446. http://dx.doi.org/10.1016/S0090-8258(03)00147-1

[34] Foxall, M.J., Barron, C.R. and Houfek, J.F. (2001) Ethnic influences on body awareness, trait anxiety, perceived risk, and breast and gynecologic cancer screening practices. Oncology Nursing Forum, 28, 727-738.

[35] Sutton, S., Saidi, G., Bickler, G. and Hunter, J. (1995) Does routine screening for breast cancer raise anxiety? Results from a three wave prospective study in England. Journal of Epidemiology and Community Health, 49, 413418. http://dx.doi.org/10.1136/jech.49.4.413

[36] Miller, L.Y. and Hailey, B.J. (1994) Cancer anxiety and breast cancer screening in African-American women: A preliminary study. Women's Health Issues, 4, 170-174. http://dx.doi.org/10.1016/S1049-3867(05)80058-1

[37] Aggarwal, A., Freund, K., Sato, A., Adams-Campbell, L.L., Lopez, A.M., Lessin, L.S., Ockene, J., Wallace, R.B., Williams, C.D. and Bonds, D.E. (2008) Are depressive symptoms associated with cancer screening and cancer stage at diagnosis among postmenopausal women? The women's health initiative observational cohort. Journal of Women's Health, 17, 1353-1361. http://dx.doi.org/10.1089/jwh.2007.0544 
[38] Friedman, L.C., Puryear, L.J., Moore, A. and Green, C.E. (2005) Breast and colorectal cancer screening among lowincome women with psychiatric disorders. Psycho-Oncology, 14, 786-791. http://dx.doi.org/10.1002/pon.906

[39] Kodl, M.M., Powell, A.A., Noorbaloochi, S., Grill, J.P., Bangerter, A.K. and Partin, M.R. (2010) Mental health, frequency of healthcare visits, and colorectal cancer screening. Medical Care, 48, 934-939. http://dx.doi.org/10.1097/MLR.0b013e3181e57901

[40] Myong, J.P., Shin, J.Y. and Kim, S.J. (2012) Factors associated with participation in colorectal cancer screening in Korea: The fourth Korean National Health and Nutrition Examination Survey (KNHANES IV). International Journal of Colorectal Disease, 27, 1061-1069.

[41] O’Donnell, S., Goldstein, B., Dimatteo, M.R., Fox, S.A., John, C.R. and Obrzut, J.E. (2010) Adherence to mammography and colorectal cancer screening in women 50-80 years of age the role of psychological distress. Women's Health Issues, 20, 343-349.

http://dx.doi.org/10.1016/j.whi.2010.04.002

[42] Peytremann-Bridevaux, I., Voellinger, R. and Santos-Eggimann, B. (2008) Healthcare and preventive services utilization of elderly Europeans with depressive symptoms. Journal of Affective Disorders, 105, 247-252. http://dx.doi.org/10.1016/j.jad.2007.04.011

[43] Xiong, G.L., Bermudes, R.A., Torres, S.N. and Hales, R.E. (2008) Use of cancer-screening services among persons with serious mental illness in Sacramento county. Psychiatric Services, 59, 929-932. http://dx.doi.org/10.1176/appi.ps.59.8.929

[44] Yee, E.F., White, R., Lee, S.J., Washington, D.L., Yano, E.M., Murata, G., Handanos, C. and Hoffman, R.M. (2011) Mental illness: Is there an association with cancer screening among women veterans? Women's Health Issues, 21, S195-S202.

http://dx.doi.org/10.1016/j.whi.2011.04.027

[45] Ludman, E.J., Ichikawa, L.E., Simon, G.E., Rohde, P., Arterburn, D., Operskalski, B.H., Linde, J.A. and Jeffery, R.W. (2010) Breast and cervical cancer screening specific effects of depression and obesity. American Journal of Preventive Medicine, 38, 303-310. http://dx.doi.org/10.1016/j.amepre.2009.10.039

[46] Tiro, J.A., Vernon, S.W., Hyslop, T. and Myers, R.E. (2005) Factorial validity and invariance of a survey measuring psychosocial correlates of colorectal cancer screening among African Americans and Caucasians. Cancer Epidemiology
Biomarkers \& Prevention, 14, 2855-2861. http://dx.doi.org/10.1158/1055-9965.EPI-05-0217

[47] Manne, S., Markowitz, A., Winawer, S., Guillem, J., Meropol, N.J., Haller, D., Jandorf, L., Rakowski, W., Babb, J. and Duncan, T. (2003) Understanding intention to undergo colonoscopy among intermediate-risk siblings of colorectal cancer patients: A test of a mediational model. Preventive Medicine, 36, 71-84. http://dx.doi.org/10.1006/pmed.2002.1122

[48] Honda, K. and Kagawa-Singer, M. (2006) Cognitive mediators linking social support networks to colorectal cancer screening adherence. Journal of Behavioral Medicine, 29, 449-460. http://dx.doi.org/10.1007/s10865-006-9068-1

[49] Rawl, S.M., Menon, U., Champion, V.L., May, F.E., Loehrer, P., Hunter, C., Azzouz, F., Monahan, P.O. and Skinner, C.S. (2005) Do benefits and barriers differ by stage of adoption for colorectal cancer screening? Health Education Research, 20, 137-148. http://dx.doi.org/10.1093/her/cyg110

[50] Sewitch, M.J., Burtin, P., Dawes, M., Yaffe, M., Snell, L., Roper, M., Zanelli, P. and Pavilanis, A. (2006) Colorectal cancer screening: Physicians' knowledge of risk assessment and guidelines, practice, and description of barriers and facilitators. Canadian Journal of Gastroenterology, 20, 713-718

[51] Zigmond, A.S. and Snaith, R.P. (1983) The hospital anxiety and depression scale. Acta Psychiatrica Scandinavica, 67, 361-370. http://dx.doi.org/10.1111/j.1600-0447.1983.tb09716.x

[52] Szklo, M. and Nieto, F. (2007) Epidemiology-Beyond the basics. 2nd Edition, Jones and Bartlett Publishers, Sudbury.

[53] Coughlin, S.S. and Thompson, T.D. (2004) Colorectal cancer screening practices among men and women in rural and nonrural areas of the United States, 1999. The Journal of Rural Health, 20, 118-124. http://dx.doi.org/10.1111/j.1748-0361.2004.tb00017.x

[54] Harewood, G.C., Wiersema, M.J. and Melton, L.J. (2002) A prospective, controlled assessment of factors influencing acceptance of screening colonoscopy. American Journal of Gastroenterology, 97, 3186-3194. http://dx.doi.org/10.1111/j.1572-0241.2002.07129.x

[55] Increased use of colorectal cancer tests-United States, 2002 and 2004. (2006) MMWR. Morbidity and Mortality Weekly Report, 55, 308-311. 